Original paper

\title{
Radiation protection of operators and patients in a hybrid Angio-MR suite
}

\author{
M. Dehairs ${ }^{\mathrm{a}, *}$, N.W. Marshall ${ }^{\mathrm{a}}, \mathrm{H}$. Bosmans $^{\mathrm{a}, \mathrm{b}}$, Martino Leghissa ${ }^{\mathrm{c}}$ \\ ${ }^{a}$ UZ Gasthuisberg, Department of Radiology, Herestraat 49, 3000 Leuven, Belgium \\ ${ }^{\mathrm{b}}$ Medical Imaging Research Centre, Medical Physics and Quality Assessment, Katholieke Universiteit Leuven, 3000 Leuven, Belgium \\ ${ }^{\mathrm{c}}$ Siemens Healthcare GmbH, 91301 Forchheim, Germany
}

\section{A R T I C L E I N F O}

\section{Keywords:}

Radioprotection

Hybrid Angio-MR

Simulation

Dose estimates

Comparative study

\begin{abstract}
A B S T R A C T
This work investigates the patient eye lens dose and x-ray scatter to the operator expected for a proposed hybrid Angio-MR concept. Two geometries were simulated for comparative assessment: a standard C-arm device for neuro-angiography applications and an innovative hybrid Angio-MR system concept, proposed by Siemens Healthineers. The latter concept is based on an over-couch x-ray tube and a detector inside an MRI system, with the aim of allowing combined, simultaneous MRI and x-ray imaging for procedures such as neurovascular interventions (including x-ray fluoroscopy and angiography imaging, 3D imaging, diffusion, and perfusion). To calculate the scattered radiation dose to the physician, Monte Carlo simulations were performed. Dose estimates of simplified models of the brain and eyes of both the patient and the physician and of the physician's torso and legs have been calculated. A number of parameters were varied in the simulation including x-ray spectrum, field of view (FOV), x-ray tube angulation, presence of shielding material and position of the physician. Additionally, 3D dose distributions were calculated in the vertical and horizontal planes in both setups. The patient eye lens dose was also calculated using a detailed voxel phantom and measured by means of thermoluminescent dosimeters (TLDs) to obtain a more accurate estimate. Assuming the same number of x-rays and the same size of the irradiated area on the patient's head, the results show a significant decrease in the scattered radiation to the physician for the Angio-MR system, while large increases, depending on setup, are expected to patient eye lens dose.
\end{abstract}

\section{Introduction}

Interventional procedures can potentially give high radiation doses to the patient and subsequently higher doses to the medical personnel can also be expected. Interventional radiologists and cardiologists are two of the most highly exposed groups of workers in hospitals in terms of ionizing radiation and have been the subject of many international and European studies [6]. This is due to the use of prolonged fluoroscopy sequences, multiple digital acquisitions and the complexity and duration of the procedures. Both the stochastic and deterministic effects of radiation are therefore of concern. For patients, skin erythema is a well-documented radiation induced effect in the catheterization laboratory and typically occurs after an absorbed skin dose of several Grays $[3,16]$. Recent studies have concluded that the eye lens is more radiosensitive than previously thought [27]. Lens opacities start developing after an acute irradiation of approximately 0.5-2 Gy, with visual impairment (cataract) developing from 5 Gy [12]. Recent studies have focused on the eye lens doses of the patient, especially for neurovascular interventions [23].
In addition, the exposure of the medical personnel performing the procedure also requires attention since staff carrying out the examinations are routinely exposed to ionizing radiation. Cataracts can develop from $150 \mathrm{mGy} /$ year for protracted exposures over many years [10]. Therefore, in 2011, the ICRP recommended the limiting eye lens dose value for medical personnel to be lowered from $150 \mathrm{mSv} /$ year to $20 \mathrm{mSv} /$ year. This has led to an urgent need for improved radiation safety and training, use of eye protection during interventional and catheterization procedures and improved occupational dosimetry $[5,7,14,15,17,26]$. Besides eye lens opacities, the prevalence of several other health hazards for occupationally exposed medical personnel has been investigated. These include: brain tumors (predominantly on the left side) [20], neurodegenerative disease [18] and a possible increase of breast cancer [8] and cardiovascular effects [18]. An extensive literature overview of papers studying the health effects of occupational dose in fluoroscopy guided interventions can be found in [24]. Great care should therefore be taken to limit the exposure to the patient, following the ALARA principle (as low as reasonably achievable), and also to protect the operators during procedures. In this respect, the

\footnotetext{
* Corresponding author.

E-mail address: michiel.dehairs@uzleuven.be (M. Dehairs).
} 
importance of teaching and training is fundamental, and should reiterate the use of the standard 'trinity' of radiation protection measures: limiting the duration of exposure, increasing the distance to the source of the (scattered) radiation and extensive use of personal protective equipment. To this end, knowledge of the operator position and orientation with respect to the scattered radiation leaving the patient can greatly help in reducing operator exposure to radiation within the x-ray room [19].

Within the EIT Health-funded P3-Stroke project, a concept for a hybrid Angio-MR has been developed, consisting of an over-couch x-ray tube and an X-ray detector inside an MRI system [13]. The Angio-MR concept is designed to perform simultaneous MRI and x-ray imaging for a wide variety of procedures [9]. Within the P3-Stroke project the Angio-MR has been investigated for neurovascular and electrophysiology (EP) procedures. The goal of this study is to determine the influence of changing from standard C-arm geometry to the setup that is being considered for this integrated Angio-MR concept on absorbed dose to the patient eye lens and the scattered dose to the primary physician for neurovascular procedures. Because the hybrid concept system tries to integrate $\mathrm{x}$-ray imaging into an MRI system, the x-ray system geometry will be different from a conventional c-arm system. Several aspects of the geometry will have a potential impact. First of all, the distances from the $\mathrm{x}$-ray source to the patient and the detector are significantly larger in the Angio-MR setup. Fig. 1 shows a graphical representation of both systems. The $\mathrm{C}$-arm is used with a typical source to image detector (SID) distance of around $105 \mathrm{~cm}$ and a source to object (SOD) distance of $75 \mathrm{~cm}$. In the current design of the Angio-MR system, the x-ray tube will be placed outside the MRI magnet and the xray detector will be incorporated in the bottom of the MRI bore. This means the SID was assumed to be $176 \mathrm{~cm}$ while the SOD will be around $136 \mathrm{~cm}$. In contrast to the conventional C-arm, the SID will not be variable, since both the source and detector are fixed in place. A second important difference is the position of the $\mathrm{x}$-ray source. The tube in the $\mathrm{C}$-arm system is usually placed in an under-couch configuration, resulting in images acquired in the Posterior-Anterior (PA) direction. The Angio-MR system, however, would use an over-couch or Anterior-Posterior (AP) configuration. The x-ray tube will be able to travel over a limited angle around the patient. This geometry has some significant implications: (1) for most neurovascular procedures, the incident $\mathrm{x}$-ray energy is first absorbed in the eye lens and then in deeper layers in the brain. This may bring the absorbed eye lens dose up to the level of peak skin dose values of standard C-arm geometries; (2) the X-rays travel through the patient first and then through the table, which increases the entrance dose to the patient by $\sim 20 \%$, in case of modern patient tables (assuming the same set of exposure parameters and the same source to patient distance in both the AP and PA direction). A final implication and possible important advantage, is that the MRI bore can act as a radiation shield for the medical personnel.

This paper presents the results of three Monte Carlo (MC) simulation sets: (1) scattered dose to the physician, (2) patient eye lens dose and (3) 3D dose distributions in vertical and horizontal planes for both system setups. Additionally, one set of TLD measurements was added to part (2) as verification of the eye lens dose simulations.

\section{Materials and methods}

\subsection{Simulating the geometries and phantoms}

The computations were performed using the PENELOPE MC code $[22,25]$. This program performs simulations of coupled electron-photon transport in arbitrary materials for a wide range of energies, from a few hundred $\mathrm{eV}$ to $1 \mathrm{GeV}$. Photon transport is simulated by means of the standard simulation scheme. For part (1) of the study, a geometry package called PENGEOM was used to model the geometries using material systems consisting of homogeneous bodies limited by quadric surfaces. After the models are created, Penelope steps in to generate the random electron-photon showers. Using this package the two geometries described above were simulated by adding a simplified phantom to represent the patient lying on the table and the primary physician standing next to the table at the femoral artery access point. In terms of organ/tissue detail, only the brain and eyes are specified for the patient phantom with the rest of the body consisting of water. The patient brain and eyes are simplified versions of the true organs and the simulations only give an indication of the effect of the changes in geometry on the absorbed energy. The phantom representing the physician also has a simplified geometric humanoid shape composed of human equivalent tissue. Eyes were included for the physician in order to investigate the influence of the MR geometry. The physician phantom does not have a brain, therefore the paper will refer to the 'head dose' as a whole. The goal was not to produce organ or effective doses with absolute accuracy, but instead to study the effect of changing geometry on the scattered radiation. Therefore, in this part of the study, the patient phantom only acts as a scatter source for the radiation reaching the primary physician. For comparative purposes, the patient brain and eye
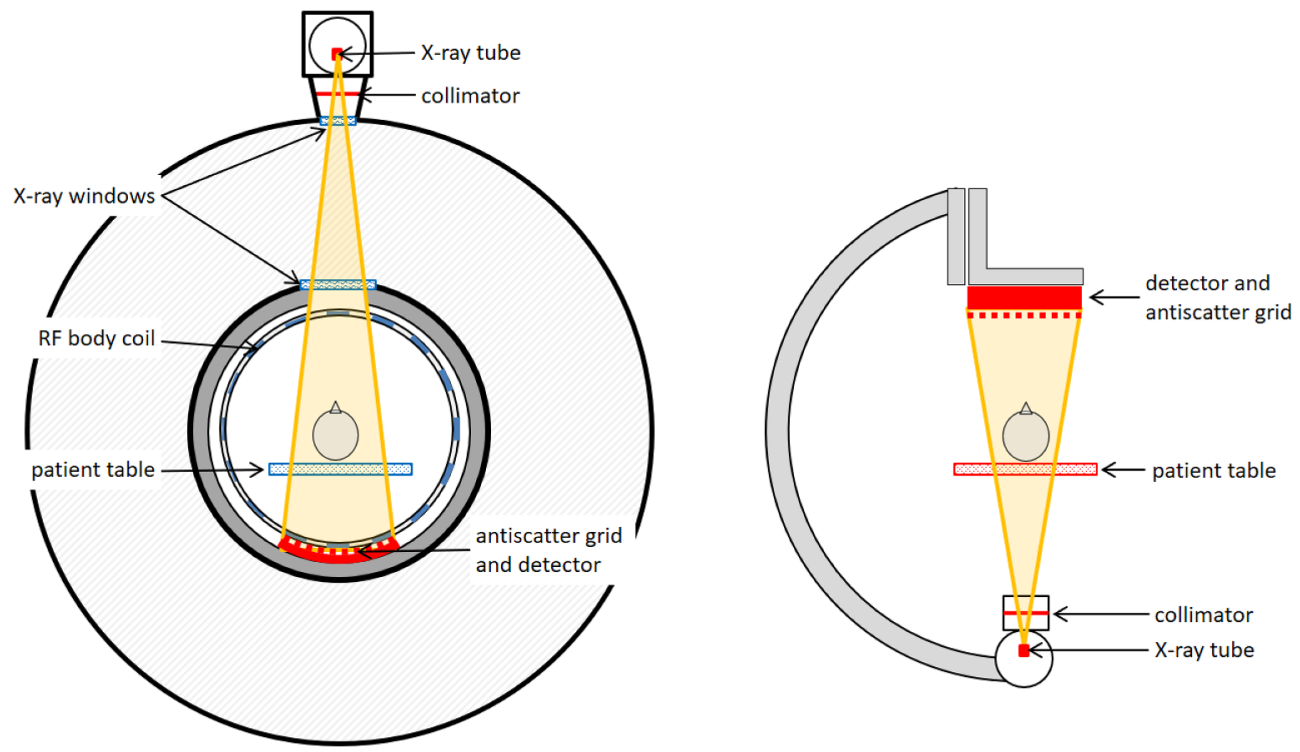

Fig. 1. Left: X-ray imaging geometry for the Angio-MR system. Right X-ray imaging geometry for a conventional C-arm angiography system (figures put to scale). 


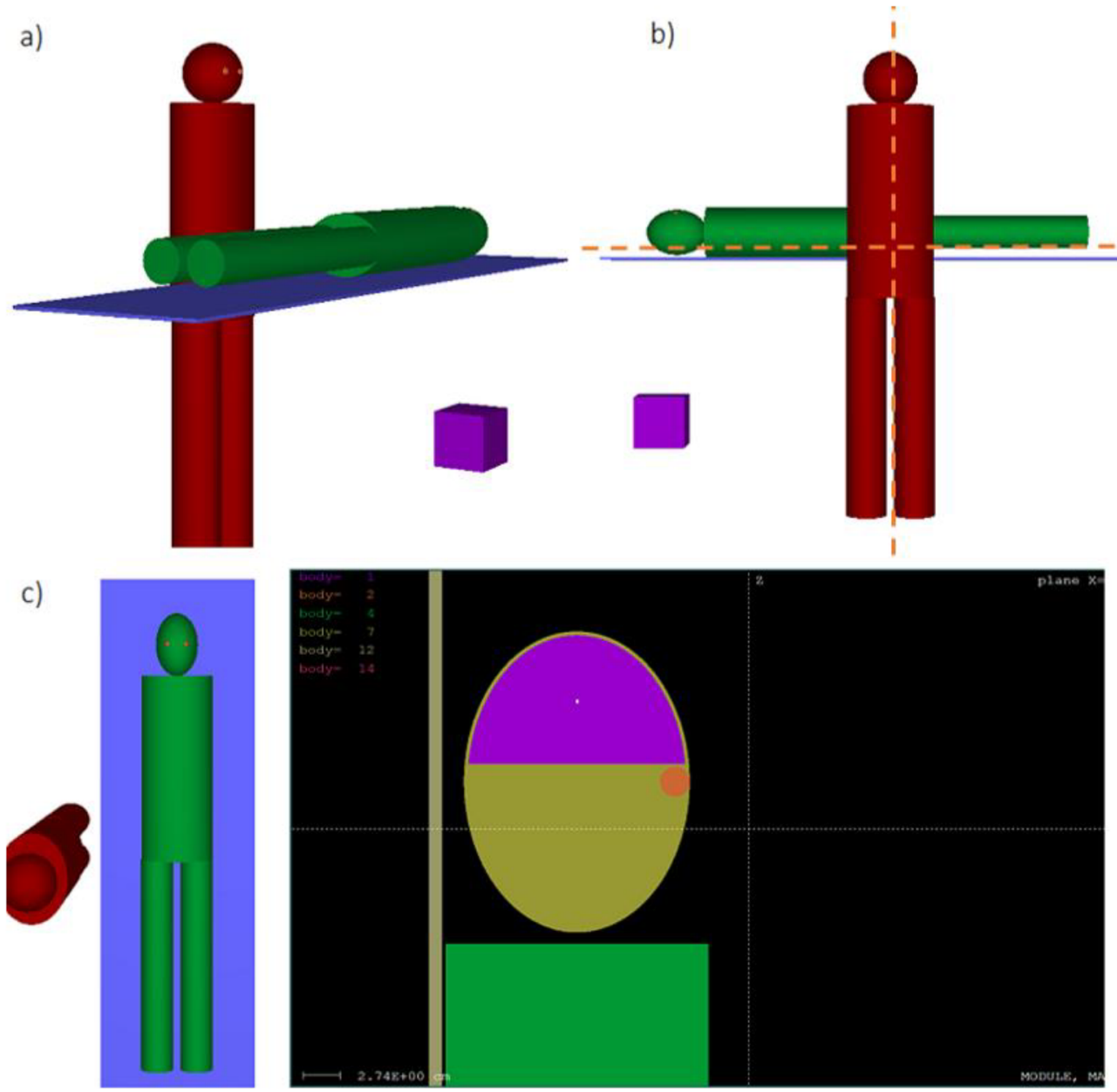

d)

Fig. 2. Graphical representation of the C-arm setup with two simplified phantoms (part 1 of the study). The purple box represents the X-ray source. a) $45^{\circ}$ view of the standard C-arm system. b) $90^{\circ}$ view. c) Top view. d) Cross section of the patient head showing the simplified brain and the right eye. Figure b) indicates the planes used for the 3D dose distribution simulations. (For interpretation of the references to color in this figure legend, the reader is referred to the web version of this article.)

dose, calculated using the quadric bodies, are also included and will be added to the results of this section. In order to make unbiased comparison between both geometries, X-ray beams were simulated such that they irradiated the same area at the incident surface of the head.

The phantoms used in part (1) of the study were built out of 3 modules (legs, torso and head) and are identical for the patient and physician. The legs and torso were cylinder shaped, with dimensions of: $32 \mathrm{~cm}$ and $25 \mathrm{~cm}$ for the torso width and depth and a height of $70 \mathrm{~cm}$, and $16 \mathrm{~cm}$ for the diameter of the leg with a length of $80 \mathrm{~cm}$. The head was elliptical with a width of $16 \mathrm{~cm}$, a depth of $18 \mathrm{~cm}$ and a height of $24 \mathrm{~cm}$. This gave a total length of $174 \mathrm{~cm}$, comparable to an average adult male. Figs. 2 and 3 visualize the system geometries and phantoms using PENGEOM in the PENELOPE code. The body of the patient in the simulations had to be defined as water while the body of the physician was defined as soft tissue, as PENELOPE can only calculate the absorbed energy in a specific material and not in a defined body part. If the same material were specified for both patient and physician, then the physician dose could not be tallied separately. Additionally, the patient is just used as a scattering medium for this particular simulation and water has comparable $\mathrm{x}$-ray interaction properties to soft tissue [1]. In order to study absorbed energy in each of the physician component regions separately, additional geometries were created in which soft (human) tissue was assigned to the region of interest (e.g. the head) and water was used for the rest (e.g. the legs and the torso). For brain and eyes, PENELOPE uses the ICRP attenuation coefficients [11]. The eyes in this part of the simulation did not contain eye lenses but instead were homogeneous spheres. The x-ray table was simulated as a thin layer of Lexan $(1 \mathrm{~cm})$, which is a polycarbonate material, as carbon fibre is not available in the PENELOPE simulation. An important remark here is that none of these materials would be suitable in an MRI environment, but since the final composition of the patient table has not yet been determined, it was decided to use the material above. The thickness was chosen such that the table attenuation was approximately $20 \%$ for a typical clinical x-ray spectrum of $80 \mathrm{kV}, 0.1 \mathrm{~mm} \mathrm{Cu}$ - the resulting attenuation was close to the measured value for one of the tables in the interventional radiology center in our hospital.

Regarding the proposed MRI system, since composition of associated components are not yet finalized, some assumptions had to be made. Based on typical MRI system designs, the following simplified material compositions were chosen. For the patient cover, body coil and other structural parts a mixture of fibreglass and epoxy was used, the gradient coil uses a combination of copper, fibreglass and epoxy and the magnet vessels (inner and outer) were created using steel. The space between the vessels was kept as air, which means that the magnet coil assembly within the vessel has been neglected for these simulations. This is a valid assumption because any $x$-ray photons scattered by the patient will only interact with the magnet vessel structure. Fig. 3e shows the MRI system without the front cover. X-rays windows are included so that the $\mathrm{x}$-rays can traverse the magnet; these are approximately equivalent to an additional $7.4 \mathrm{~mm}$ of aluminum. The shielding in both systems was simulated using lead glass for the ceiling mounted radio protective shields ( $0.5 \mathrm{~mm}$ lead equivalent) and lead for 

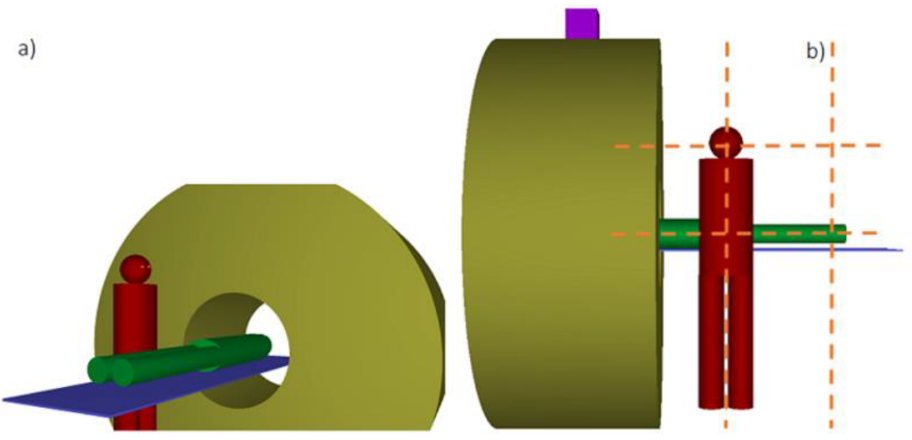

c)
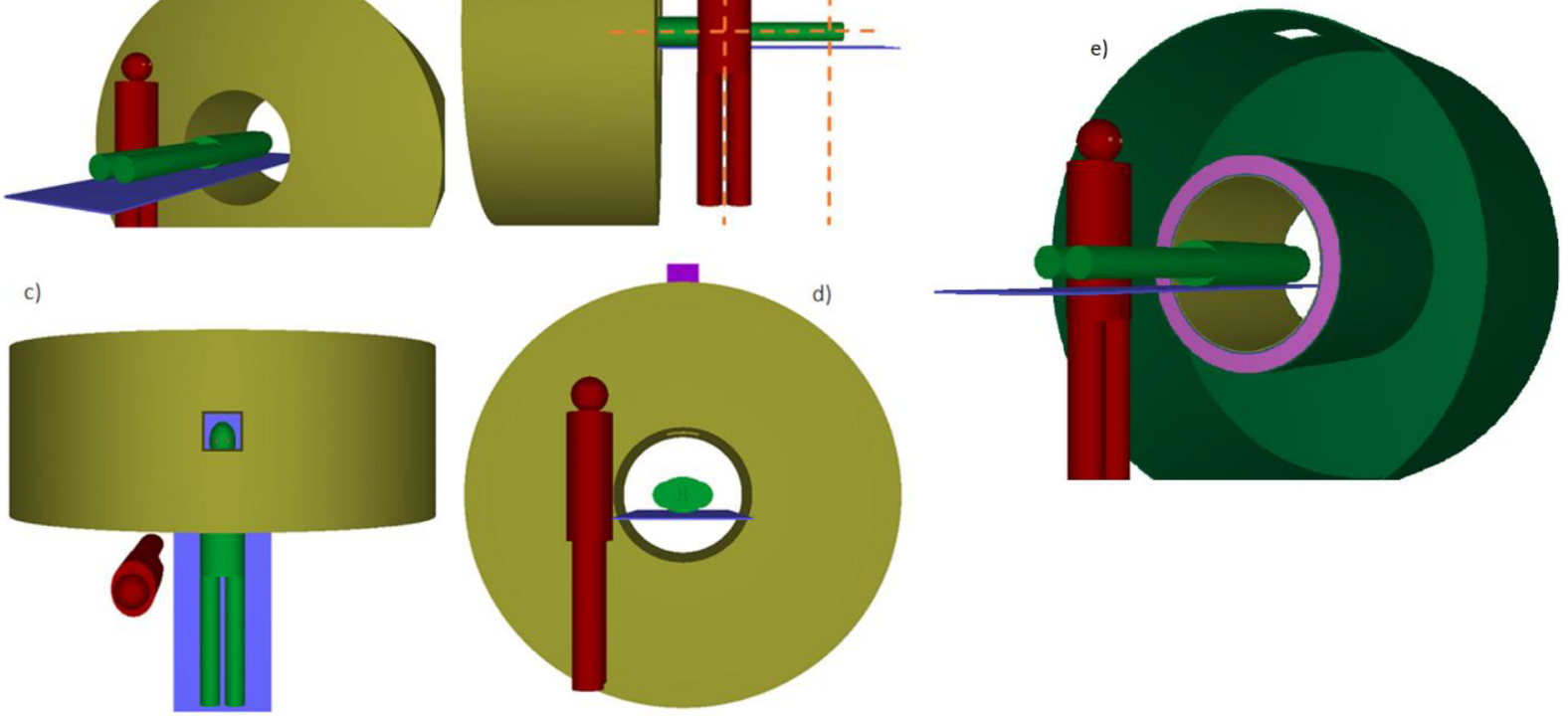

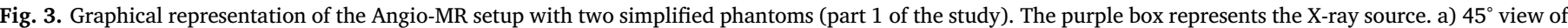

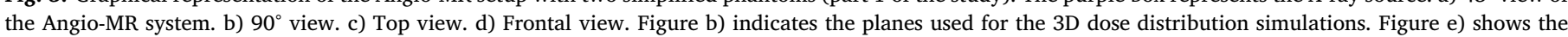
system without front cover. (For interpretation of the references to color in this figure legend, the reader is referred to the web version of this article.)

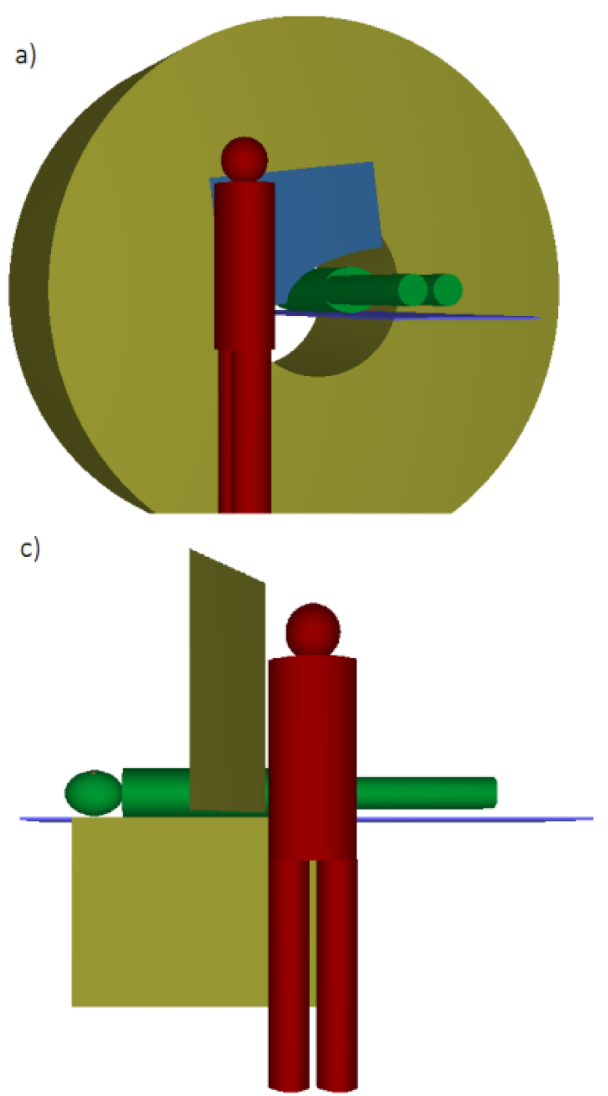

b)
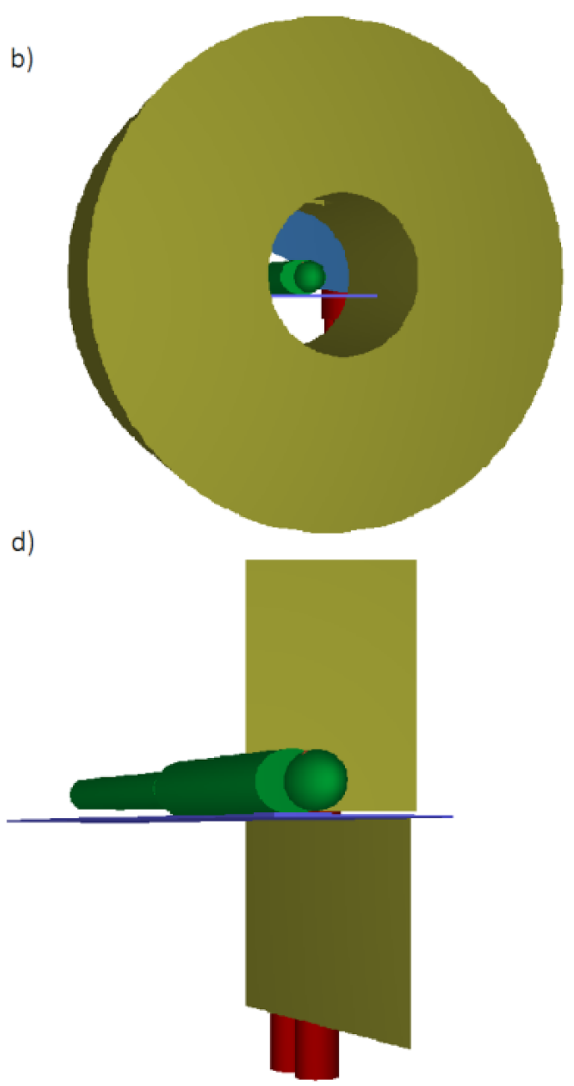

Fig. 4. Graphical representation of the shielding of both setups (part 1 of the study). a) $45^{\circ}$ view of the Angio-MR system. b) Back view of the Angio-MR system c) $90^{\circ}$ view of the C-arm system. d) Back view of the C-arm system. Note: the same color of the MRI system (top) and the lead shields (bottom) does not indicate they are made of the same material. the table mounted radio protective curtain $(1 \mathrm{~mm})$ (Fig. 4). Shielding through a lead apron or lead glasses was not included in this study.

In part (2) of the study, the Zubal MRI adult head voxel phantom was used in order to study patient eye lens dose in greater detail [28].
Fig. 5 shows a graphical representation of one of the slices of the voxel phantom: the eye lenses are clearly visible. The voxels have dimensions of $0.11 \mathrm{~mm}, 0.11 \mathrm{~mm}$ and $0.14 \mathrm{~mm}$ in the $\mathrm{x}, \mathrm{y}$ and $\mathrm{z}$ directions respectively. Each voxel is indexed with a number that designates a given 


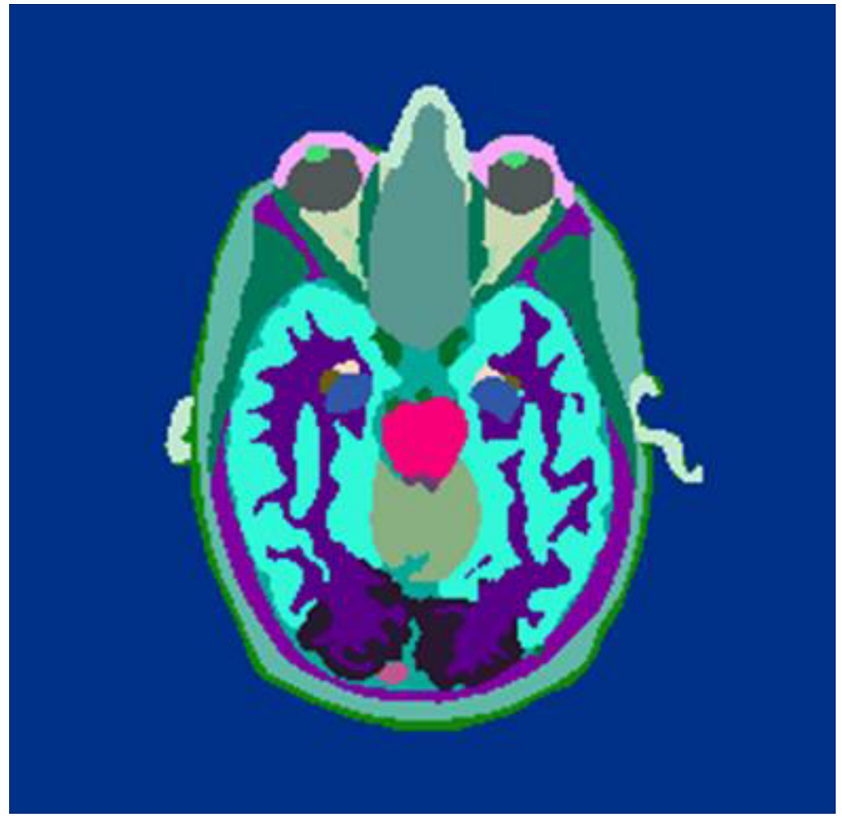

Fig. 5. Slice of the ZUBAL head voxel phantom (used in part 2 of the study). Color coding was used to help visualize the different organs. The eye lens is visible as the small light green ellipses below the pink border at the top of the picture. Voxels have dimensions of $(0.11,0.11$ and $0.14 \mathrm{~mm})$ in the $(\mathrm{x}, \mathrm{y}$ and $\mathrm{z})$ direction. (For interpretation of the references to color in this figure legend, the reader is referred to the web version of this article.)

organ or internal structure, which is then linked to the MC data needed to perform the simulation (density, attenuation coefficients etc.). This phantom was then loaded into the PENELOPE software and irradiated in the AP and PA direction to investigate the effect on the eye lens dose. In this simulation the table was excluded as the exact composition of the patient table was not known. To verify the simulations, TLD measurements were performed using the head of the CIRS ATOM phantom irradiated in the same geometry as in the simulations [2] (Fig. 6). Only the teenage CIRS phantom was available, however there is only a small difference in head size between this phantom and the Zubal. Two TLDs were used per eye, making four in total per irradiation. Additionally, bismuth eye shields were placed on the TLDs to see their efficiency in lowering the eye lens dose [4]. In order to eliminate the effect of distance, measurements in the AP and PA direction were corrected such that the distance from the TLDs to the X-ray source was identical. This was done because the long distances proposed for the Angio-MR system geometry could not be reproduced.

These simulations all examine energy absorbed by the different materials, more specifically the whole body of the physician and the eyes lens of the patient. In part (3) of this study additional sets of simulations were performed in order to see the 3 dimensional dose distribution at the (theoretical) location of the primary physician, for both systems. A volume of air of $200 \times 200 \times 200 \mathrm{~cm}^{3}$ was defined on the physician side of the table, adjacent to the table, at the position where the MRI bore ends. This volume was split into cubes of $10 \times 10 \times 10 \mathrm{~cm}^{3}$, defining the resolution of the 3D distribution. The dose distribution was then calculated for horizontal and vertical planes of $10 \mathrm{~cm}$ thick, defined in this volume. These simulations were performed using approximately $2 \times 10^{9}$ photons, since the amount of energy deposited in air is low (approximately $12 \mathrm{~h}$ simulation run time on a 2017 XPS Dell laptop using an Intel $^{\oplus}$ Core $^{\mathrm{TM}}$ i7-770HQ CPU @ $2.8 \mathrm{GHz}$ and $8 \mathrm{~GB}$ of RAM).

\subsection{Overview of the simulations}

For part 1, the following simulation parameters were varied in order to study their effect on the physician scatter radiation dose:

- Several x-ray spectra were used: $80 \mathrm{kVp}$ with $0.1 \mathrm{~mm} \mathrm{Cu}$ filtration, $80 \mathrm{kVp}$ with $7.4 \mathrm{~mm} \mathrm{Al}$ filtration and then the same filtrations but with $100 \mathrm{kVp}$ (4 in total). The $80 \mathrm{kVp}(0.1 \mathrm{~mm} \mathrm{Cu})$ spectrum is a typical setting for a $\mathrm{C}$-arm system during neurological procedures and was chosen as the reference for the conventional system.

- Two circular FOVs were chosen: a small FOV with a diameter of $10 \mathrm{~cm}$ and a large FOV with a diameter of $16 \mathrm{~cm}$ (both specified at the entrance plane to the head). The beams are always centered on the center of the brain, with the consequence that for the small FOV the eyes were not directly irradiated, while they are irradiated with the large FOV.

- Two projections were used: the $0^{\circ}$ angulation defines the PA projection in the case of the C-arm system, while for the Angio-MR system this defines the AP projection. The second angulation is at $90^{\circ}$ with the source in both cases located at the left side of the patient body giving the Right Lateral (RLAT) projection (angulations in the cranio-caudal direction are not foreseen in the proposed AngioMR system.

- The length of the MRI bore was varied between $100 \mathrm{~cm}$ and $120 \mathrm{~cm}$. - The standard simulation places the physician in both geometries at
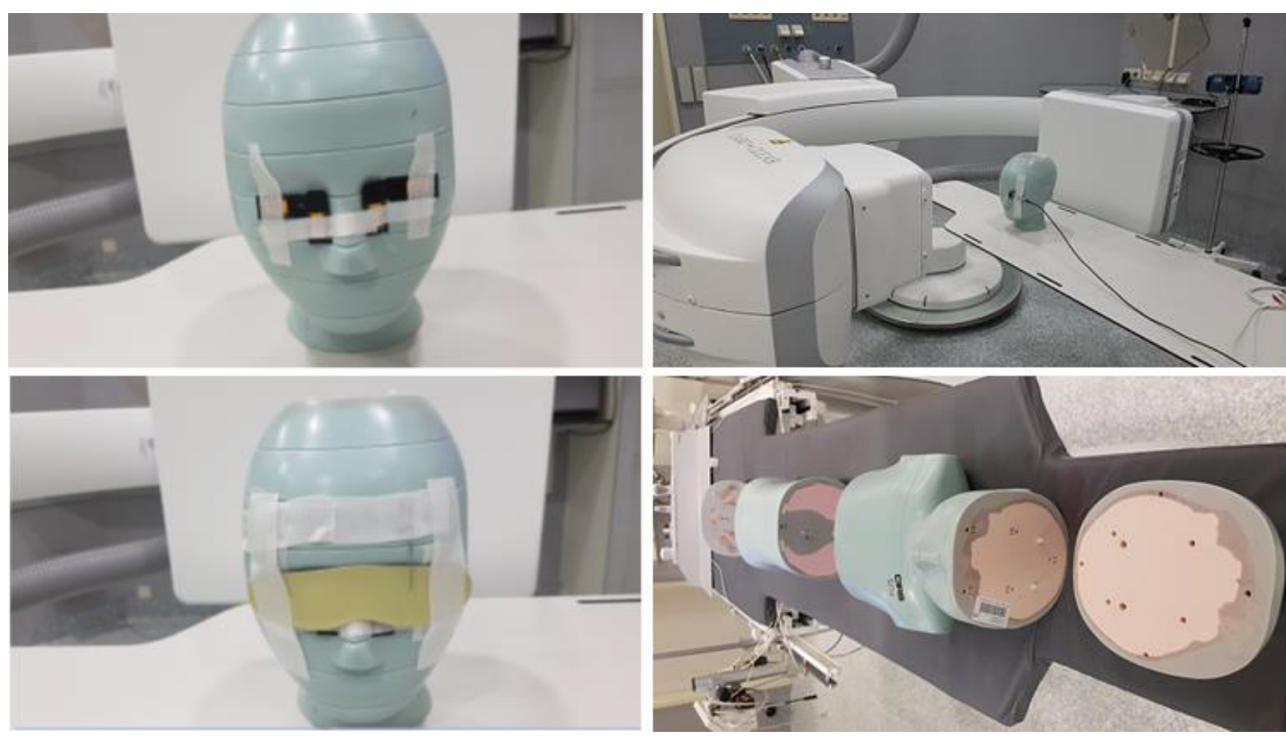

Fig. 6. Setup of the TLD measurements using the head of the CIRS Atom phantom (part 2 of the study). The head was irradiated in the AP and PA direction without the table in the beam to match the simulations (top right). Two TLDs were used per eye (top left) and additionally, a Bismuth eye shield was used to see its effect on the eye lens dose (bottom left). The CIRS phantom consists out of different slices made from tissue equivalent materials (bottom right). 
identical positions. To see the potential shielding effect of the MRI, the physician was placed a small step back $(20 \mathrm{~cm}$ further away from the table and $20 \mathrm{~cm}$ closer to the MRI).

- The standard C-arm setup was repeated, adding a ceiling mounted lead glass shield to compare the shielding effect to that of the MRI system. Shield thickness was equivalent to $0.5 \mathrm{~mm}$ lead, a typical value in interventional radiology suites. Additionally a table mounted lead curtain was added ( $1 \mathrm{~mm}$ lead equivalent) to simulate full shielding of the physician.

- A ceiling mounted lead glass shield was also added in the Angio-MR setup.

For part 2, the irradiation of the patient head (Zubal voxel phantom) was varied between PA and AP projections at $0^{\circ}$ angulation and at $45^{\circ}$ angulation (Angio-MR: right posterior oblique (RPO), C-arm: right anterior oblique (RAO)). The $80 \mathrm{kVp}$ and $0.1 \mathrm{~mm} \mathrm{Cu} \mathrm{x}$-ray spectrum was used for the measurements. Two rectangular FOVs were chosen: the small FOV coronal view covered the head from the top of the brain until $1 \mathrm{~cm}$ above the eyes, while the large FOV coronal view reached $2 \mathrm{~cm}$ below the eyes.

Each of these simulations was performed using at least $1 \times 10^{8}$ photons to limit the error on the results while also keeping simulation time acceptable $(\sim 1 \mathrm{~h})$. Because the goal of the simulations was not to calculate absolute organ doses, the results of the simulations are given as relative values with the conventional $\mathrm{C}$-arm results as the reference value.

\section{Results}

Table 1 compares absorbed energy to the physician for the AngioMR and the conventional C-arm set up for the first set of simulations, examining scattered radiation. This table contains results for two angulations, two FOVs and two X-ray spectra. The absorbed energy by the physician (dose estimate) was examined for the individual body segments to see the direction of the highest fraction of scattered $\mathrm{x}$-rays (towards the head, torso or legs). As the amount of radiation absorbed by the different segments depends on their dimension, the volume percentage of the different body segments is important and has the following values: $7 \%, 55 \%$ and $38 \%$ for the head, torso and legs respectively. Table 2 gives the results after implementation of one of the following dose reduction techniques, examined in this study: using lead shielding (ceiling and/or table mounted), positioning behind the MRI (outside the X-ray scatter field) and using a longer MRI bore. Table 3 shows the effect of different X-ray filtrations on the absorbed energies. A filtration of $7.4 \mathrm{~mm} \mathrm{Al}$ was used instead of $0.1 \mathrm{~mm} \mathrm{Cu}$ as this is likely to be the filtration used in the Angio-MR system. For the whole body physician dose the confidence interval ( 2 standard deviations) is $\sim 1 \%$ of the total absolute value, however uncertainty was larger for the physician eye dose. For the results without any shielding (values around 1 ) the 2 standard deviation value is $\sim 5 \%$ of the total absolute value, however in case of any shielding (low values found in Tables 2 and 3) the uncertainty is much larger, at $50-100 \%$ of the absolute value, because the amount of scatter radiation reaching such a small target is very small. To reduce this uncertainty by a factor of 10 , to reach a similar uncertainty as with the other absorbed energies, would require the simulation time to increase by a factor of 100 .

Table 4 contains the results of the patient eye lens dose simulations, calculated using the Zubal voxel phantom. Results are given for two FOVs, two angulations and four X-ray spectra. The ratio of the energy in the AP direction and the PA direction is also calculated. Table 5 shows the results of the TLD measurements. Measurements in the AP direction were repeated using the bismuth eye shield. The absorbed energy values for the $0^{\circ}$ measurements are corrected such that they represent the same measured source to eye distance. For the $45^{\circ}$ angle, a distance correction was made for each eye separately, to account for the influence of distance. The results in Table 4 are not corrected for distance, since a uniform parallel X-ray beam was used, so there is no reduction in $\mathrm{x}$-ray dose as a function of distance. This was specifically chosen in order not to have an influence of distance on the simulations. The simulations at $45^{\circ}$ do not calculate the eye lens dose in each eye separately while the TLD measurements make this distinction and thus cannot be compared with the TLD measurements.

In addition to the absorbed energies, further simulations were performed to visualize the 3 dimensional dose distributions, in a region adjacent to the imaging systems (Figs. 7 and 8). These figures show the absorbed dose in air in units of $\mathrm{eV} / \mathrm{g} /$ photon for $10 \mathrm{~cm}$ thick planes. The coordinate system is centered on the patient brain, which in turn is positioned at the isocentre of the system in both geometries. Fig. 7 gives results of the dose distribution for the vertical planes shown in Figs. $2 \mathrm{~b}$ and $3 \mathrm{~b}$. For this figure, the $\mathrm{x}$-axis therefore represents the distance away from the table. The values start at $30 \mathrm{~cm}$ since the table is $60 \mathrm{~cm}$ wide (i.e. the edge of the table is at $+30 \mathrm{~cm}$ ). Note that the numbers on the $\mathrm{x}$ axis start at $20 \mathrm{~cm}$, but the first values of the figures start at $\mathrm{x}=30 \mathrm{~cm}$. The y-axis represents the height from the floor. The floor begins at the position of $-100 \mathrm{~cm}$, with the patient table defined at $-10 \mathrm{~cm}$ height and the head of the physician starting at $150 \mathrm{~cm}$ above the floor. The

Table 1

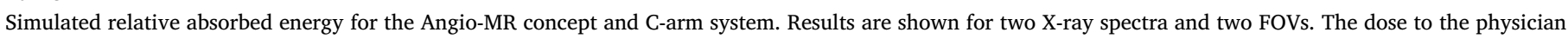

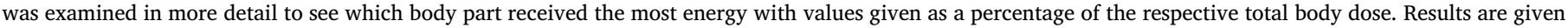
relative to the $\mathrm{C}$-arm $0^{\circ}, 80 \mathrm{kV}$ values for both the small and large FOV separately.

Simulated relative absorbed energy for the Angio-MR and C-arm system (i.e. relative to C-arm $0^{\circ}, 80 \mathrm{kV}$ )

\begin{tabular}{|c|c|c|c|c|c|c|c|c|}
\hline Spectrum: $80 \mathrm{kVp}(0.1 \mathrm{~mm} \mathrm{Cu})$ & Small FOV & & & & Large FOI & & & \\
\hline Organ: & $\mathrm{C}$-arm $0^{\circ}$ & $\mathrm{C}-\operatorname{arm} 90^{\circ}$ & Angio-MR $0^{\circ}$ & Angio-MR $90^{\circ}$ & $\mathrm{C}-\operatorname{arm} 0^{\circ}$ & $\mathrm{C}$-arm $90^{\circ}$ & Angio-MR $0^{\circ}$ & Angio-MR $90^{\circ}$ \\
\hline Brain patient & 1 & 1.23 & 1.24 & 1.25 & 1 & 1.30 & 1.25 & 1.35 \\
\hline Eye patient & 1 & 1.49 & 4.34 & 1.45 & 1 & 2.87 & 23.52 & 2.21 \\
\hline Body physician: & 1 & 0.42 & 0.40 & 0.26 & 1 & 0.43 & 0.50 & 0.27 \\
\hline Head & $3.0 \%$ & $7.3 \%$ & $2.5 \%$ & $3.5 \%$ & $3.4 \%$ & $7.8 \%$ & $3.1 \%$ & $3.8 \%$ \\
\hline Torso & $37.2 \%$ & $45.9 \%$ & $84.8 \%$ & $76.7 \%$ & $39.3 \%$ & $44.7 \%$ & $84.2 \%$ & $74.9 \%$ \\
\hline Legs & $59.8 \%$ & $46.8 \%$ & $12.7 \%$ & $19.8 \%$ & $57.3 \%$ & $47.5 \%$ & $12.7 \%$ & $21.3 \%$ \\
\hline Eye physician & 1 & 1.01 & 0.97 & 0.80 & 1 & 1.01 & 1.14 & 0.71 \\
\hline \multicolumn{9}{|l|}{ Spectrum: $100 \mathrm{kVp}(0.1 \mathrm{~mm} \mathrm{Cu})$} \\
\hline Brain patient & 1.02 & 1.24 & 1.25 & 1.25 & 1.02 & 1.31 & 1.27 & 1.36 \\
\hline Eye patient & 1.21 & 1.68 & 4.49 & 1.63 & 1.21 & 3.04 & 22.71 & 2.36 \\
\hline Body physician: & 1.14 & 0.53 & 0.51 & 0.35 & 1.12 & 0.53 & 0.61 & 0.36 \\
\hline Eye physician & 1.24 & 1.81 & 1.25 & 0.84 & 1.09 & 1.23 & 1.43 & 0.86 \\
\hline
\end{tabular}


Table 2

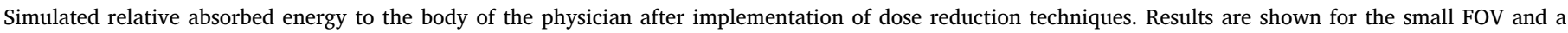
spectrum of $80 \mathrm{kVp}$ and $0.1 \mathrm{~mm}$ Cu. Results are given relative to the C-arm $0^{\circ}, 80 \mathrm{kV}$ values of Table 1 .

\begin{tabular}{|c|c|c|c|c|c|c|}
\hline \multicolumn{7}{|c|}{ Simulated relative absorbed energy after implementation of dose reduction techniques (i.e. relative to C-arm $0^{\circ}, 80 \mathrm{kV}$ ) } \\
\hline \multirow[t]{2}{*}{ Spectrum: $80 \mathrm{kVp}(0.1 \mathrm{~mm} \mathrm{Cu})$} & \multicolumn{6}{|l|}{ Small FOV } \\
\hline & MRI step back $0^{\circ}$ & MRI step back $90^{\circ}$ & MRI Longer bore $0^{\circ}$ & MRI Longer Bore $90^{\circ}$ & MRI with Shield $0^{\circ}$ & MRI with Shield $90^{\circ}$ \\
\hline Body physician: & 0.03 & 0.03 & 0.21 & 0.14 & 0.10 & 0.09 \\
\hline \multirow[t]{2}{*}{ Eye physician } & 0.02 & 0.03 & 0.06 & 0.10 & 0.02 & 0.03 \\
\hline & $\begin{array}{l}\text { C-arm with ceiling } \\
\text { shield } 0^{\circ}\end{array}$ & $\begin{array}{l}\text { C-arm with ceiling } \\
\text { shield } 90^{\circ}\end{array}$ & $\begin{array}{l}\text { C-arm with table } \\
\text { shield } 0^{\circ}\end{array}$ & $\begin{array}{l}\text { C-arm with table } \\
\text { shield } 90^{\circ}\end{array}$ & $\begin{array}{l}\text { C-arm with both } \\
\text { shields } 0^{\circ}\end{array}$ & $\begin{array}{l}\text { C-arm with both } \\
\text { shields } 90^{\circ}\end{array}$ \\
\hline Body physician: & 0.69 & 0.23 & 0.35 & 0.21 & 0.04 & 0.03 \\
\hline Eye physician & 0.07 & 0.06 & 1.05 & 1.01 & 0.08 & 0.05 \\
\hline
\end{tabular}

Table 3

Simulated relative absorbed energy for estimated Angio-MR specific X-ray spectra. These simulations use a different amount of filtration: $7.4 \mathrm{~mm} \mathrm{Al}$ instead of $0.1 \mathrm{~mm} \mathrm{Cu}$. Results are given relative to the C-arm $0^{\circ}, 80 \mathrm{kV}$ values of Table 1 .

\begin{tabular}{lllll}
\hline $\begin{array}{l}\text { Simulated relative absorbed energy for Angio-MR specific X-ray spectra (i.e. relative to } \\
\left.\text { C-arm } 0^{\circ}, 80 \mathrm{kV}\right)\end{array}$ & \multicolumn{3}{l}{} \\
\hline $\begin{array}{l}\text { Spectrum: } 80 \mathrm{kVp} \\
(7.4 \mathrm{~mm} \mathrm{Al})\end{array}$ & Small FOV & & \\
\hline Organ: & Angio-MR & Angio-MR $0^{\circ}$ & Angio-MR & Angio-MR $90^{\circ}$ \\
& $0^{\circ}$ & step back & $90^{\circ}$ & step back \\
\hline Brain patient & 1.24 & 1.24 & 1.24 & 1.24 \\
Eye patient & 4.51 & 4.51 & 1.59 & 1.59 \\
Body physician & 0.46 & 0.04 & 0.31 & 0.04 \\
Eye physician & 0.96 & 0.03 & 0.80 & 0.04 \\
\hline Spectrum: $100 \mathrm{kVp}$ & & & & \\
$\quad(7.4$ mm Al) & & & & \\
\hline Brain patient & 1.26 & 1.26 & 1.26 & 1.26 \\
Eye patient & 4.63 & 4.63 & 1.76 & 1.76 \\
Body physician & 0.57 & 0.05 & 0.39 & 0.05 \\
Eye physician & 1.26 & 0.04 & 1.07 & 0.09 \\
\hline
\end{tabular}

vertical planes are $10 \mathrm{~cm}$ thick and are centered on the physician for both systems (7a and 7c) and $50 \mathrm{~cm}$ further towards the patient feet in the Angio-MR system concept (7b). Fig. 8 represents the results for the horizontal planes also shown in Figs. $2 \mathrm{~b}$ and $3 \mathrm{~b}$. These planes are again $10 \mathrm{~cm}$ thick and are positioned just above the patient table for both systems ( $8 \mathrm{a}$ and $8 \mathrm{c}$ ) and between 160 and $170 \mathrm{~cm}$ height for the AngioMR system concept (approximate height of the physician eyes) (8b). For this figure the $\mathrm{x}$-axis again shows the distance away from the table and the $y$-axis shows the distance away from the end of the MRI bore in the head-feet direction of the patient, therefore this number starts at $+50 \mathrm{~cm}$, since the MRI is $100 \mathrm{~cm}$ long. In order to make a fair comparison, the $\mathrm{y}$ axis for the conventional C-arm (Fig. 8c) also starts at $+50 \mathrm{~cm}$. Note that the numbers on the y-axis start at $40 \mathrm{~cm}$, but the first values of the figures start at $y=50 \mathrm{~cm}$.

\section{Discussion}

The first part of this study investigated the scatter radiation to the primary physician. The results in Table 1 show a decrease in the physician whole body dose when using the MR setup for all different simulation parameters. In this simulation the physician is standing $10 \mathrm{~cm}$ from the table at the hip of the patient, for both geometries. At this location, the physician using the MR setup, absorbed about half the energy as the physician in the C-arm setup. Looking at the dose distributions of Figs. 7 and 8, it is clear that the MRI bore acts as an effective radiation shield. Not only are the lower legs and the head shielded (if the physician is sufficiently tall, the exact height where this occurs is position dependent), but almost no radiation reaches when the operator is standing far enough from the table (in the direction perpendicular to the table length). Fig. 7a shows that the dose drops close to zero when standing at $70 \mathrm{~cm}$ from the center of the table, or in this simulation: $40 \mathrm{~cm}$ from the table edge, and above $160 \mathrm{~cm}$ (this follows from the geometry used in this simulation which may not be identical in the final system). The effect of the over-couch versus under-couch configuration becomes clear when looking at the absorbed energy of the separate body parts. Since most of the scattered radiation impinging on the operators originates from the incident plane of the x-ray beam (i.e. via backscattered radiation), the legs in the conventional (under-couch) $\mathrm{C}$-arm setup receive much higher radiation exposure than in the AngioMR setup. This can be seen as an advantage for the conventional C-arm, since the lower extremities are generally of lower radio sensitivity (except for the reproductive organs, which are well protected by the

Table 4

Simulated absorbed energy in the eye lens. Results are shown for four different X-ray spectra and are given relative to the $\mathrm{C}$-arm $0^{\circ}$, $80 \mathrm{kV}$ value.

\begin{tabular}{|c|c|c|c|c|c|c|}
\hline \multicolumn{7}{|c|}{ Simulated relative absorbed energy in the eye lens (i.e. relative to C-arm $0^{\circ}, 80 \mathrm{kV}$ ) } \\
\hline \multirow[t]{2}{*}{ X-ray spectrum } & \multicolumn{3}{|l|}{ Small FOV } & \multicolumn{3}{|l|}{ Large FOV } \\
\hline & C-arm $\left(\mathrm{PA}-0^{\circ}\right)$ & Angio-MR (AP-0 $\left.0^{\circ}\right)$ & Ratio (AP/PA) & C-arm $\left(\mathrm{PA}-0^{\circ}\right)$ & Angio-MR (AP- $0^{\circ}$ ) & Ratio (AP/PA) \\
\hline $80 \mathrm{kVp}(0.1 \mathrm{~mm} \mathrm{Cu})$ & 1 & 4.2 & 4.2 & 2.2 & 101.3 & 46.0 \\
\hline $80 \mathrm{kVp}(7.4 \mathrm{~mm} \mathrm{Al})$ & 1.1 & 5 & 4.5 & 2.5 & 95.4 & 38.2 \\
\hline $100 \mathrm{kVp}(0.1 \mathrm{~mm} \mathrm{Cu})$ & 1.4 & 5.2 & 3.7 & 2.8 & 97.9 & 34.9 \\
\hline \multirow[t]{2}{*}{$100 \mathrm{kVp}(7.4 \mathrm{~mm} \mathrm{Al})$} & 1.5 & 5.5 & 3.7 & 3.2 & 93.3 & 29.2 \\
\hline & C-arm (RA0-45 $)$ & Angio-MR (RPO-45) & Ratio (RPO/RAO) & C-arm (RA0-45) & Angio-MR (RPO- $45^{\circ}$ ) & Ratio (RPO/RAO) \\
\hline $80 \mathrm{kVp}(0.1 \mathrm{~mm} \mathrm{Cu})$ & 1.5 & 2.7 & 1.8 & 11.6 & 62.2 & 5.4 \\
\hline
\end{tabular}


Table 5

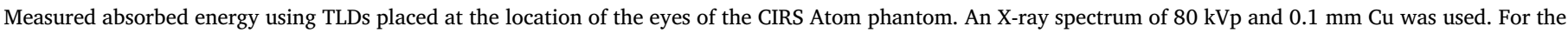

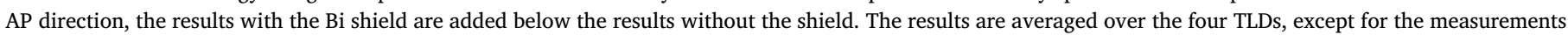

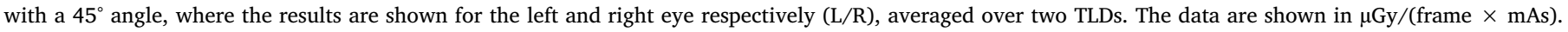

Measured Dose using TLDs

\begin{tabular}{|c|c|c|c|c|c|c|c|c|}
\hline \multicolumn{3}{|l|}{ Small FOV } & \multicolumn{6}{|l|}{ Large FOV } \\
\hline AP projection $0^{\circ}$ & PA projection $0^{\circ}$ & Ratio (AP/PA) & AP projection $0^{\circ}$ & PA projection $0^{\circ}$ & Ratio (AP/PA) & $\mathrm{RP0}$ projection $45^{\circ}$ & RA0 projection $45^{\circ}$ & Ratio (RPO/RAO) \\
\hline 3.2 & 0.8 & 4.0 & 95.8 & 2.8 & 34.2 & $105 / 104$ & $12.3 / 3.2$ & $8.5 / 32.4$ \\
\hline With Bi shield & \multicolumn{2}{|c|}{ Ratio (without/with) } & With Bi shield & \multicolumn{2}{|c|}{ Ratio (without/with) } & With Bi shield & Ratio (without/with) & \\
\hline 3.4 & \multicolumn{2}{|l|}{0.95} & 32.2 & \multicolumn{2}{|l|}{2.98} & $36.9 / 22.8$ & $2.9 / 4.6$ & \\
\hline
\end{tabular}

lead apron) and the eyes are far from the scattered radiation. Due to the over-couch configuration in the Angio-MR setup, most of the X-rays will scatter towards the upper torso and the head of the physician, but a fraction of the X-rays are shielded by the MRI itself. The net effect depends of course on the height and position of the physician. For radiation protection purposes, this can be important, as the Angio-MR system can potentially lower the eye lens dose of the physician. Our simulations indicate that lead glasses may not need to be worn. Using $7.4 \mathrm{~mm} \mathrm{Al}$ as the filtration in the Angio-MR system seems to increase the while body dose by approximately $10 \%$ in all cases (see Table 3 ). This could be caused by the slightly higher average energy of photons in the $7.4 \mathrm{~mm} \mathrm{Al}$ spectra.

The results in Table 2 show how the dose to the physician could be lowered by using a number of dose saving techniques. Using the proper shielding and standing at the optimal position have always been important ways of lowering personnel dose. However with the Angio-MR system, these could have an even greater impact. Adding a ceiling mounted shield to the MRI system lowered the absorbed energy by a factor of 4 for the $0^{\circ}$ angulation. The effectiveness of this shield results again from the over-couch configuration of the X-ray source and that the greatest intensity of scattered radiation is backscattered towards the source/upwards towards the body of the physician. A table mounted curtain could have been simulated to shield against forward scattered $\mathrm{x}$ rays towards the lower half of the physician. However a curtain hanging down from the table side (i.e. along the table length) would not provide shielding from x-rays originating from the patients head. An efficient table-based shield for the MRI system would probably have curtains hanging orthogonally from the table, across the entire width of the table, however this setup was not studied. As for the MRI system, the table mounted lead shield in the C-arm setup stopped the majority of the X-rays compared to the ceiling suspended shield. Of course, in good clinical practice, both shields should be correctly and actively used. When using both shields in the conventional system, the absorbed energy drops to a lower value than the MR setup with the ceiling mounted shield. However, an additional simulation was performed where the physician in the MR setup took one small step back i.e. $20 \mathrm{~cm}$ further away from the table and $20 \mathrm{~cm}$ closer to the MRI, in order to shield himself from the scattered radiation. Fig. 9 gives a graphical representation of this scenario. This action dramatically decreases the physician dose to a value similar as the conventional system setup with both shields. However this would also mean the physician would no longer have direct visual contact with the patient. In the MRI world, this is routine practice and many cameras are installed. They could address these needs. Whether this is possible in clinical practice will have to be investigated in clinical studies.

This scenario did not have a radiation shield, but by combining both the radiation shield and taking a safe position, the radiation dose could be lowered even further. As mentioned before, the physician eye dose can be lowered significantly in the Angio-MR setup if the physician is standing in the optimal position. There are some additional parameters such as physician height that also play a role, but in any case, using the proper shielding and position can minimize the eye dose. Even though this cannot be considered a dose reduction technique, the length of the MRI bore was also increased from $100 \mathrm{~cm}$ to $120 \mathrm{~cm}$. This was done because the final dimensions of the Angio-MR were not fixed at the time of simulation. As expected, using a longer bore length reduces the physician dose, since the window of the X-rays for reaching the physician has become even smaller. The results show a decrease by a factor of 2 for both angulations. Both the longer and shorter bore MRI units will have pros and cons, however. As the simulations show, the longer bore would decrease the dose towards the medical personnel. Also, designing a magnet, with a homogeneous magnetic field would be easier with a longer bore. A shorter MRI bore would however give the medical personnel easier access to the patient, a safety aspect that cannot be ignored for interventional procedures.

Changing the FOV or the X-ray spectrum did not significantly change the results of the comparison between both system geometries. Changing the size of the patient however, could potentially have a larger effect. In most cases, the diameter of the patient's head does not scale proportionally with patient weight and thus we expect less variation in adult head diameter as for the abdomen, for example. In this study only neurological procedures were investigated and therefore the body of the patient is not directly irradiated and acts as an absorber of scattered radiation coming from the head. This means that an increase in abdomen diameter could reduce the dose to the physician, in both setups, assuming the head size is similar for the thicker patient. In the MR setup, since the exit window of the scattered X-rays is already limited, increasing the size of the patient significantly reduces the amount of X-rays that can reach the physician. This also happens for the conventional C-arm setup, but the effect is smaller.

Tables 4 and 5 show the increase in eye lens dose the Angio-MR setup, assuming the eyes are at the same distance from the X-ray source. Of course, the distance in the MR setup will be much greater which could reduce the relative eye lens dose, assuming the same exposure factors are used. Because the exact acquisition parameters of the hybrid concept system are not known, distance was taken out of the comparison. Even when not irradiating the eyes directly, the eye lens dose in the Angio-MR setup is approximately a factor of 4 higher than in the conventional under-couch situation, since the intensity of scatter radiation will be more intense at the entrance point of the head than at the exit point. When comparing the ratios in Tables 4 and 5, the results from the simulations closely match the TLD measurements. The results for the larger FOV, where the eyes are located in the primary X-ray beam, show an increase of a factor between 29 and 46, depending on the X-ray spectrum. Averaged over these values, the dose increased a factor of $\sim 37$ in the MR system. In comparison, the TLD measurements show an increase of approximately a factor of 34 . This is of course a significant increase, meaning extra care needs to be taken to make sure each exposure to the patient is justified. Radiation protection techniques for the eyes have to be considered when possible, these include: collimating the X-ray beam to exclude the eyes, tilting the head forward to move the eyes out of the primary FOV and using protective eye shields for the patient. The main contribution should however come from the combined use of (3D) MR angiography and x-ray imaging, or 

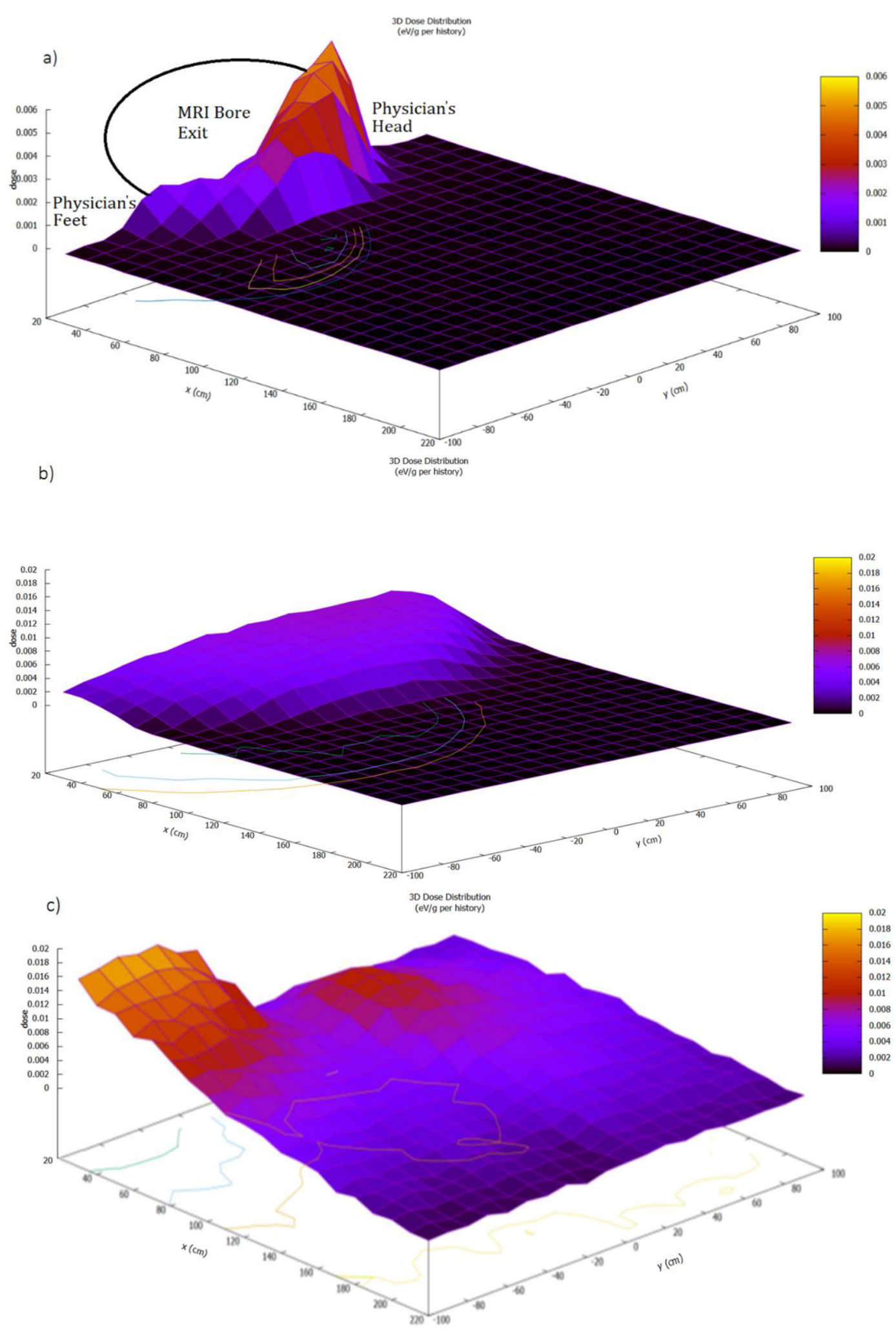

Fig. 7. Dose distribution defined in vertical planes in the MRI geometry and C-arm geometry. a) Plane at the center of the physician for the Angio-MR geometry, b) plane $50 \mathrm{~cm}$ further than the physician center, and c) plane at the center of the physician for the C-arm geometry. The position of the physician's head and feet are indicated in the top figure. See Figs. $2 \mathrm{~b}$ and $3 \mathrm{~b}$ to help visualize the planes.

any other new technique such as image fusion with input of other modalities. It may be possible that MR angiography series could replace some of the x-ray acquisitions and therefor the physician dose may not be a large or determining factor in the doses from the hybrid system. An important remark however is that by changing the angulation of the projections, the disadvantage of the MR geometry starts to disappear. At $90^{\circ}$, the position of the tube is identical for both systems, meaning the eye dose will be similar. Thus, using larger angulations could help in reducing the eye lens dose.

The results at the bottom of Table 5 show the effect of using the 

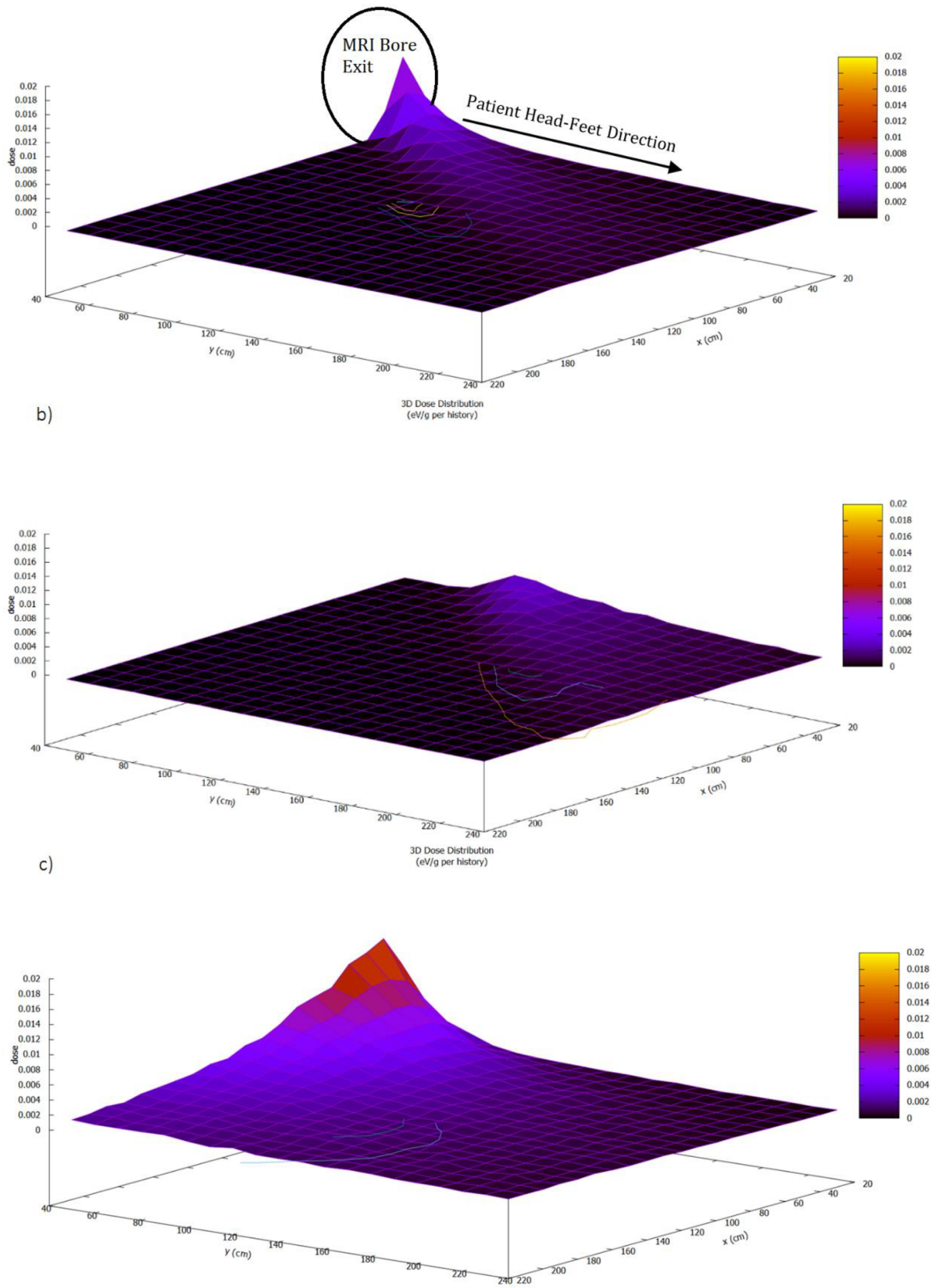

Fig. 8. Dose distribution defined in horizontal planes in the MRI geometry and C-arm geometry. a) Plane just above the patient table for the Angio-MR geometry, b) plane between 160 and $170 \mathrm{~cm}$ height, and c) plane just above the patient table for the C-arm geometry. The position of the patient is indicated in the top figure. See Figs. $2 \mathrm{~b}$ and $3 \mathrm{~b}$ to help visualize the planes.

bismuth eye shield. The ratio (without/with) is less than 1.0 for the small FOV, indicating the shield offers no advantage or may even be detrimental for the small FOV. Whether the Bismuth shield is actually detrimental to the eye dose when the eyes are not directly irradiated depends on the scattered radiation distribution. Potentially, there could be multiple scattering of $\mathrm{x}$-rays (e.g. from the forehead towards the eye shield and then be scattered back towards the eyes), but we expect that this would be rare. It is more likely that the uncertainty associated with the measured TLD explains the ratio of 0.95 that was found. Turning to the the large FOV $\left(0^{\circ}\right)$, the dose was reduced by a factor of 3 . Whether this shield can be used in clinical practice should be investigated further though, since this may limit the visibility of vessels behind the eyes, 

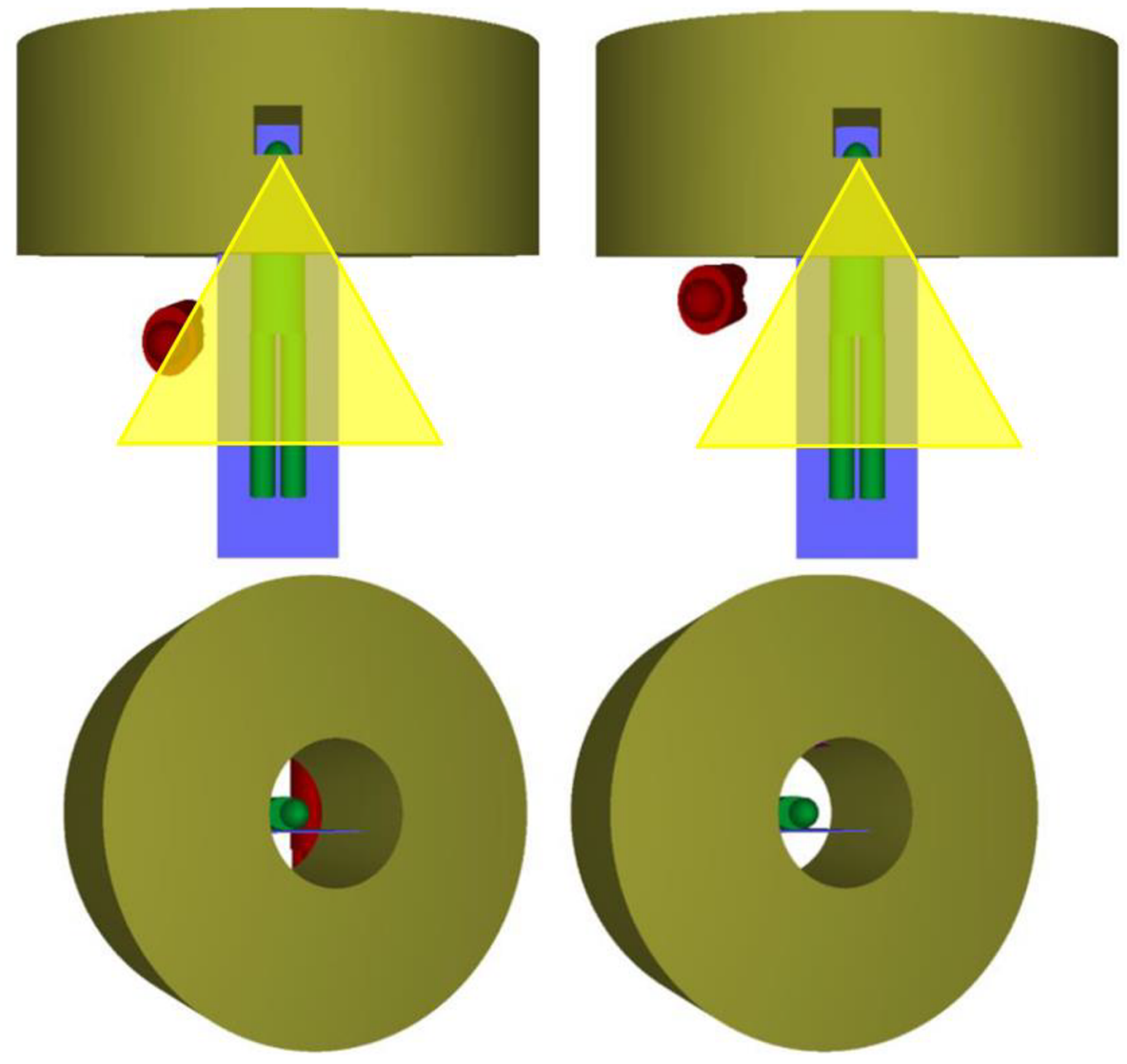

Fig. 9. This figure shows the effect of the physician taking a step back. Part of the scatter radiation is indicated with the yellow triangle The figures on the left show the 'normal' position of the physician (standing next to the table and at patient's hips). The figures on the right show the physician standing $20 \mathrm{~cm}$ further away from the table and closer to the MRI system. The figures indicate that the physician was partly standing in the FOV of the scattered X-ray beam, while taking a small step back and closer to the MRI can completely hide the physician from the Xrays. (For interpretation of the references to color in this figure legend, the reader is referred to the web version of this article.) depending on the shield, and it may not be easy to (re)position the shields when the patient is already in the magnet. A recent study has, however shown that the use of specialized eye protectors can considerably reduce the patient's eye lens dose during neuro-interventional procedures [21]. They found an eye lens reduction of $62 \%$ (consistent with our factor of 3 ) and that this protector does not significantly perturb the fluoroscopy image and was completely invisible on the acquisition image due to image subtraction. The same reasoning applies for using collimation. If the vessels of interest are located behind the eyes, which is often the case (example for the circle of Willis and arteria cerebri media), this technique cannot be used. In that case, tilting the patient's head forward might be the only option to lower the eye lens dose, but again this might not always be a viable option if patient mobility is hindered in the magnet. An important remark on the eye lens dose results is that these simulations and measurements do not include the patient table. As discussed before, this immediately increases the entrance dose for the Angio-MR system by approximately $20-30 \%$, depending on the angulation (this could be even larger for very steep angulations).

To get an idea of the absolute eye lens dose magnitude expected, a dose survey was performed on 60 patients that underwent a thrombectomy procedure in the past year at our hospital. This was done retrospectively by using the dose monitoring platform DOSE (Qaelum NV, Leuven, Belgium). This platform can calculate the peak skin dose (PSD) immediately after the procedure is performed and uses different patient models to distinguish between different patient sizes and body parts (in this case; head). The PSD gives an indication of what the eye lens could be in a worst case scenario. The average PSD for these 60 patients was $0.26 \mathrm{~Gy}$ with a maximum value of $1.97 \mathrm{~Gy}$. The values for the 25th percentile, the median and 75th percentile are: $0.11,0.21$ and 0.28 Gy respectively. In total, four patients had a PSD above $0.5 \mathrm{~Gy}$. Since the threshold of developing lens opacities starts at 0.5 Gy (12), these values show that protecting the patient eyes when possible should be encouraged.

Some remarks regarding the over-couch irradiation of the patient: the reason why this system has to use an over-couch configuration is because installation of the x-ray source underneath the magnet is considered to be not practical due to space constraints. Since the X-rays will have to come from above (when a frontal view is required), one way of avoiding the eyes being irradiated first could be to simply place the patient on their stomach so the X-rays are incident on the back of the patient head. Whether this is a potential option will depend on the patient and will have to be discussed between the physician and patient if possible.

\section{Conclusion}

This study used the Monte Carlo program PENELOPE to investigate the eye lens dose of the patient and the whole body dose of the primary physician for two different interventional radiology systems: a hybrid Angio-MR concept system and a conventional C-arm system. The computations show that for the MRI system the dose to the eyes significantly increases due to the over-couch configuration. Therefore, extra measures may have to be taken to limit eye dose, for example by exploiting the combined MR plus x-ray imaging opportunities, and/or to make sure the eyes are not irradiated by the primary X-ray beam or by shielding the eyes when possible.

For the physician it is clear that the shielding by the MRI can strongly reduce the dose from scattered radiation and is comparable to using lead glass shields in the conventional setup. Positioning behind/ to the side of the MRI bore during exposures can be considered an active form of radiation protection and reduces the absorbed dose to a point close to zero. The results also show that placing a ceiling mounted lead shield above the patient should probably still be considered as it can drastically lower the scattered dose to the physician, for positions close to the patient. Depending on the physician's ability to make use of 
the MRI's shielding, a lead apron could perhaps be thinner than in a conventional room. Additionally, the wearing of a thyroid shield and lead glasses is still recommended, especially as, for over-couch configurations, much of the scattered X-ray radiation exits the patient in the direction of the physician's head. The simulations did however show that even for an average sized physician standing at the normal position and with a short MRI bore length, most of the X-rays travelling towards the head are stopped, reducing physician eye lens dose.

\section{Acknowledgement}

This study was performed as part of the P3 stroke project. P3-Stroke is an innovation project supported by EIT Health, a network of best-inclass health innovators that collaborates across borders and delivers solutions to enable European citizens to live longer, healthier lives. EIT Health is supported by the EIT, a body of the European Union. (eithealth.eu).

\section{References}

[1] Akar A, et al. Measurement of attenuation coefficients for bone, muscle, fat and water at 140, 364 and $662 \mathrm{keV} \gamma$-ray energies. J Quant Spectrosc Radiat Transfer 2006;102:203-11.

[2] Atom Dosimetry Phantom: 900 Asbury Ave Norfolk, Virginia 23513, USA, Tel: 757855-2765, WWW.CIRSINC.COM.

[3] Balter S, Hopewell JW, Miller DL, et al. Fluoroscopically guided interventional procedures: a review of radiation effects on patients' skin and hair. Radiology 2010;254:326-41.

[4] Barrier Technologies: Secure Shield, CT eye shield, 3017 Greene Street, Hollywood, Florida, USA, www.barriertechnologies.com.

[5] Borrego D, Kitahara CM, Balter S. Yoder C occupational doses to medical staff performing or assisting with fluoroscopically guided interventional procedures. Radiology 2020;294:353-9.

[6] Chida $\mathrm{K}$, et al. Occupational dose in interventional radiology procedures. Am J Roentgenol 2013 Jan;200(1):138-41. https://doi.org/10.2214/AJR.11.8455.

[7] Cornacchia S, Errico R, La Tegola L, et al. The new lens dose limit: implication for occupational radiation protection. Radiol Med 2019;124:728-35. https://doi.org/ 10.1007/s11547-019-01027-7.

[8] Doody MM, Freedman DM, Alexander BH, et al. Breast cancer incidence in U.S Radiologic technologists. Cancer 2006;106:2707-15.

[9] Fahrig R, et al. Design, performance, and applications of a hybrid X-Ray/MR system for interventional guidance. Proc IEEE 2008;96(3).

[10] ICRP. Nonstochastic effects of ionizing radiation. ICRP Publication 41. Ann. ICRP
$1984 ; 14: 3$.

[11] ICRP. Basic Anatomical and physiological data for use in radiological protection reference values. ICRP Publication 89. Ann. ICRP 2002;32:3-4.

[12] ICRP. Recommendations of the international commission on radiological protection. ICRP Publication 103. Ann. ICRP 2007;37:2-4.

[13] Leghissa Martino, Rebecca Fahrig, Simon James Calvert, Adrian Mark Thomas. Medical imaging system comprising a magnet unit and a radiation unit, WO2018095587A1, https://patents.google.com/patent/WO2018095587A1/en.

[14] Matsubara K, Lertsuwunseri V, Srimahachota S, Anchali Krisanachinda A, Tulvatana W, Khambhiphant B, Sudchai W, Madan Rehani M. Eye lens dosimetry and the study on radiation cataract in interventional cardiologists. Physica Med $2017 ; 44: 232-5$

[15] Medici S, Pitzschke A, Cherbuin N, Boldini M, Sans-Merce M, Damet J. Eye lens radiation exposure of the medical staff performing interventional urology procedures with an over-couch X-ray tube. Physica Med 2017;43:140-7.

[16] Mahesh Mahadevappa. Radiation dose management for fluoroscopically guided interventional medical procedures. Med Phys 2012;39:5789-90. https://doi.org/ 10.1118/1.4747450. (NCRP Report No. 168).

[17] Marie Nowak, et al. Eye lens monitoring programme for medical staff involved in fluoroscopy guided interventional procedures in Switzerland. Physica Med 2019;57:33-40.

[18] Picano E, Vano E, Domenici L, et al. Cancer and non-cancer brain and eye effects of chronic low-dose ionizing radiation exposure. BMC Cancer 2012;12:157-69.

[19] Principi S, Farah J, Ferrari P, Carinou E, Clairand I, Ginjaume M. The influence of operator position, height and body orientation on eye lens dose in interventional radiology and cardiology: Monte Carlo simulations versus realistic clinical measurements. Physica Med 2016;32:1111-7.

[20] Roguin A, Goldstein J, Bar O, et al. Brain and neck tumors among physicians performing interventional procedures. Am J Cardiol 2013;111:1368-72.

[21] Safari MJ, et al. A method to reduce patient's eye lens dose in neuro-interventional radiology procedures. Radiat Phys Chem 2016;125:75-81.

[22] Salvat Francesc A. Code system for monte carlo simulation of electron and Facultat de Fisica (ECM), Universitat de Barcelona Spain, OECD; 2015. PENELOPE- 2014 Photon Transport.

[23] Sánchez RM, et al. Radiation doses in patient eye lenses during interventional neuroradiology procedures. AJNR Am J Neuroradiol 2016;37(3):402-7.

[24] Seulki Ko MD, et al. Health effects from occupational radiation exposure among fluoroscopy-guided interventional medical workers: a systematic review. J Vasc Interv Radiol 2018;29(3):353-66.

[25] Sempau J, Acosta E, Baro J, Fernandez-Varea JM, Salvat F. An algorithm for Monte Carlo simulation of the coupled electron-photon transport. Nucl Instrum Methods B 1997;132:377-90.

[26] Stahl CM, Meisinger QC, Andre MP, Kinney TB, Newton IG. Radiation risk to the fluoroscopy operator and staff. AJR 2016;207:737-44.

[27] Vano E, Kleiman NJ, Duran A, et al. Radiation-associated lens opacities in catheterization personnel: results of a survey and direct assessments. J Vasc Interv Radiol 2013;24:197-204.

[28] Zubal IG, Harrell CR, Smith EO, Rattner Z, Gindi G, Hoffer PB. Computerized threedimensional segmented human anatomy. Med Phys 1994;21(2):299-302. 